\title{
Sobre la vigencia del Manifiesto de Bauru en tiempos adversos: anotaciones sobre la demolición del cuidado a la salud mental en Brasil
}

\author{
Sobre a vigência do Manifesto de Bauru em tempos adversos: \\ anotações sobre a demolição do cuidado à saúde mental no \\ Brasil
}

On the validity of the Bauru Manifesto in adverse times: notes on the demolition of mental health care in Brazil

\section{Manuel Desviat*}

\begin{abstract}
Resumen - La reforma de Brasil va a confirmar tanto la importancia de la sustancial modificación de la atención a la salud mental como la fragilidad de las construcciones sociales que pretenden la universalidad e igualdad de las prestaciones. De la importancia de lo primero, da testimonio el cierre de muchos de los manicomios y la creación de recursos más eficientes, pero, sobre todo, la construcción de un discurso ético y técnico progresista, inmerso en un amplio movimiento en defensa de los derechos humanos del loco y el diferente. De lo segundo, da cuenta el desmonte de los servicios públicos que viene sucediendo en los últimos años en Brasil, y que forma parte del retroceso, en este caso, brutal, de las políticas públicas, propiciado por la hegemonía neoliberal a nivel internacional. Mas los tiempos adversos, de resistencia, sirven para reconstruir saberes y practicas. A luta continua.
\end{abstract}

Palabras clave: reforma psiquiátrica en Brasil; retroceso de la atención psicosocial; crisis de la salud publica en el neoliberalismo.

Resumo - A reforma psiquiátrica do Brasil confirma, por uma parte, a importância da substancial modificação da atenção à saúde mental, como, por outra, a fragilidade das construções sociais que pretendem a universalidade e a igualdade de seu acesso. A reforma psiquiátrica dá testemunho do fechamento de muitos manicômios e da criação de recursos mais eficientes, mas, sobretudo, da construção de um discurso ético e técnico progressista, imerso em um amplo movimento em defesa dos direitos humanos do louco e do diferente. No que concerne à sua fragilidade, observa-se o "desmonte" dos serviços públicos e que forma parte do retrocesso, neste caso, brutal, das políticas públicas, propiciado pela hegemonia neoliberal, em nível internacional. Mas, os tempos adversos, de resistência, servem para reconstruir saberes e práticas. A luta continua.

* Psiquiatra, ex asesor de la Organización Panamericana de Salud (OPS) para Brasil. E-mail: desviatm@gmail.com. ORCID: https://orcid.org/0000-0002-3942-4690. 
Palavras-chave: reforma psiquiátrica no Brasil; retrocesso da atenção psicossocial; crise da saúde pública no neoliberalismo.

\begin{abstract}
The Brazilian psychiatric reform will confirm both the importance of the substantial modification of mental health care and the fragility of social constructions that claim universality and equality of benefits. The importance of the first is witnessed by the closure of many of the asylums and the creation of more efficient resources, but, above all, by the construction of a progressive ethical and technical discourse, in the context of a broader movement in defense of the human rights of the crazy and the different. As for the second, it is accounted for by the dismantling of public services that has been happening in recent years in Brazil, and which is part of the decline, brutal in this case, of public policies, caused by neoliberal hegemony at the international level. But the adverse times, of resistance, serve to reconstruct knowledge and practices. The struggle goes on.

Keywords: psychiatric reform in Brazil; decline in psychosocial care; public health crisis in neoliberalism.
\end{abstract}

\title{
La fragilidad de lo común
}

La historia no se cansa de mostrarnos como las conquistas sociales son siempre provisionales. Mucho más cuando, como en el caso de la reforma psiquiátrica brasileña, (antimanicomial, desinstitucionalizadora, de salud mental colectiva), cuestiona tanto la hegemonía privatizadora del neoliberalismo, como la propia estructura político-económica que predispone o causa el sufrimiento psíquico, daño colateral o precio necesario del desarrollo del capitalismo. Parece cada vez más evidente que la apuesta democrática y social estorba al neocapitalismo.

Se juega con el mito de la mejor eficacia de los mercados y el necesario adelgazamiento de las cuentas públicas, cuando la toma de los gobiernos nacionales por el capital financiero no supone el adelgazamiento del gasto público (consideremos el gasto en las fuerzas armadas o de seguridad, entre otros), sino la venta del erario a los fondos buitres internacionales, hospitales, pensiones, universidades. Supone, sí, la acumulación ilimitada del capital, como previó Marx y el aumento exponencial de las desigualdades (DESVIAT, 2019).

En el proyecto socialdemócrata, tras la Segunda Guerra Mundial, la salud, la educación, las prestaciones sociales (el Estado del bienestar), quisieron ser una política de Estado, un pacto social que trascendiera la alternancia democrática de los partidos en los gobiernos. Pero, para el fundamentalismo neoliberal, una vez dueño del mundo, tras la caída del muro de Berlín, las leyes sociales surgidas tras la crisis de 1929 y la catástrofe de la Segunda Guerra Mundial, son un obstáculo, un residuo a suprimir, como lo son las políticas sociales de algunos estados latinoamericanos 


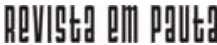

\} SOBRE LA VIGENCIA DEL MANIFIESTO - DESVIAT, M. \}

DOI: $10.12957 /$ REP.2022.63530

iniciadas a contracorriente en las últimas décadas, siendo las medidas públicas emprendidas en Brasil uno de los principales objetivos por abatir.

Ahí tenemos el impeachment de Dilma Rousseff, la condena fraudulenta de Lula y la culminación del golpe de Estado con el acceso al poder de la extrema derecha representada por Jair Bolsonaro. Esta vez no fueron necesarios los tanques ni los escuadrones de la muerte entrenados por la CIA, bastó con el Banco Mundial, la deuda y la corrupción política, mediática y judicial. Los objetivos se cumplieron, dejando un país devastado: privatización de empresas estratégicas, demolición de las políticas públicas, retirada de los derechos de los trabajadores, desempleo, hostigamiento de los pueblos originarios, permisividad de la deforestación amazónica, degradación, en definitiva, las instituciones y los valores democráticos. Una política de por si criminal que se agudiza durante la pandemia con el negacionismo genocida del presidente de la nación.

En la sanidad, una enmienda constitucional (95/216) va a congelar durante 20 años el gasto publico destinado a diversas políticas sociales, entre otras el Sistema Único de Salud (SUS), lo que está repercutiendo de forma inmediata en un progresivo deterioro de la sanidad pública.

Nos encontramos en un escenario ya visto, cuyo primer ensayo fue Chile tras el golpe de Estado al gobierno de la Unidad Popular: un escenario perfectamente diseñado por los grandes lobbies financieros, el Banco Mundial, el Fondo Monetario Internacional, la reserva federal dos Estados Unidos y los especialistas pagados por la administración estadunidense en un encuentro que se llamó "Consenso de Washington". Los fines quedaron claros: desregulación absoluta de la economía, ausencia total de reglas, subordinación de los Estados a los mercados y la reducción drástica del gasto público. Se trata de un programa en el cual la privatización de la salud y de las pensiones se convierte en objetivo principal.

\section{La demolición de la política nacional de atención a la salud mental brasileña}

En 2017, la Comissão Intergestores Tripartites (CIT), instancia de articulación y pacto nacional del Sistema Único de Saúde (SUS - Sistema Único de Salud), inicia las mudanzas normativas que ponen fin a la política brasileña de Salud Mental - una política que había situado a la reforma psiquiátrica a la vanguardia de las políticas desinstitucionalizadoras y de salud mental comunitaria en América Latina. La CIT va a establecer las nuevas directrices para el funcionamiento de la red de atención psicosocial (Raps), reformulando el diseño, el funcionamiento, la metodología de evaluación de los servicios y la orientación clínica, reglamentando, en definitiva, las nuevas políticas de salud mental. Nueva política esa que permite la creación de nuevos hospitales psiquiátricos, en contra de la ley 


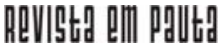

\} SOBRE LA VIGENCIA DEL MANIFIESTO - DESVIAT, M. \}

DOI: $10.12957 /$ REP.2022.63530

federal de 2011, y retira la posibilidad de pasar el dinero que ocasiona el ingreso hospitalario a los servicios de residencia terapéutica.

En un artículo para la revista Trabalho, Educação e Saúde, investigadores del Instituto de Psiquiatria da Universidade do Rio de Janeiro analizan los documentos ministeriales promulgados de 2016 a 2019, donde se plasma la Nova Política Nacional de Saúde Mental (PNSM), en la que se vuelve a incentivar los ingresos psiquiátricos en instituciones totales y se estanca la implantación de recursos comunitarios. En su estudio los autores destacan la ausencia de debate con profesionales e investigadores del campo de la atención psicosocial en su elaboración y la ignorancia de instancia legales como Consejo Nacional de Salud, así como las conclusiones de las cuatro Conferencias Nacionales de Salud Mental, y toda la legislación nacional e internacional que ampara el modelo de la reforma llevado a cabo durante tres décadas. Por otra parte, denuncian que lo que viene siendo designado por el gobierno como "nueva" PNSM está siendo impuesto sin ninguna legitimidad social ni política ni sustento legal alguno (CRUZ; GONÇALVES; DELGADO, 2020).

En el entendimiento de la radicalidad de este empeño en el desmonte de la reforma psicosocial hay que tener en cuenta que el gobierno no está solo. Desde sus inicios hubo grandes resistencias entre los poderes conservadores, en especial en buena parte del poder profesional. La Asociación Brasileña de Psiquiatría siempre fue resistente a la reforma, defendiendo la necesidad de los hospitales psiquiátricos no solo en el debate profesional sino también en amplias campañas mediáticas de cara a la población. Por otra parte, hay que recalcar que la reforma se hizo a contracorriente de la política sociosanitaria impuesta a nivel mundial por el capital, lo que se pone de manifiesto en la ambivalencia y las concesiones a la derecha por parte de los gobiernos socialdemócratas en todo el mundo, como sucedió en Brasil durante la gestión de Dilma con el nombramiento de un Ministro de Salud y un Coordinador Nacional de Salud Mental contrarios a la reforma sanitaria y psiquiátrica, así como el financiamiento de las comunidades terapéuticas para personas con un uso problemático del uso de alcohol y otras drogas, auténticos manicomios privados, desconectados de los servicios sanitarios y de salud mental, con un abordaje prohibicionista y punitivo.

\section{Aportes técnicos, éticos y políticos a la reforma brasileña}

Hay dos acontecimientos en el horizonte de la democratización del país tras la dictadura militar que van a constituir el armazón teórico y práctico, los pilares de la reforma brasileña: el Manifiesto de Bauru (1987) y la II Conferencia Nacional de Salud Mental (1992). Tales acontecimientos se inscriben en el proceso de Reforma sanitaria (implantación del SUS) y 
están presentes los procesos de reforma psiquiátrica ya iniciados en otros países, leídos desde la cultura salubrista de la Salud Colectiva, impulsada en Brasil por el Centro Brasileiro de Estudos da Saúde (Cebes) y la Associação Brasileira de Saúde Coletiva (Abrasco), que va introducir el debate en el campo de las políticas sociales de la salud y las obligaciones del Estado al respecto (RESENDE CARVALHO, 2007; FAGUNDES, 2006).

El Manifiesto, carta fundacional del Movimento Antimanicomial, supone un desafío radicalmente nuevo al afirmar la lucha del Movimento dos Trabalhadores en Saúde Mental no solo en el cierre de los hospitales psiquiátricos y la creación de redes comunitarias, sino en la lucha por la igualdad, por los derechos de la clase trabajadora, por hacer frente al racismo, por la defensa de los pueblos indígenas y de las mujeres.

Nossa atitude marca uma ruptura. Ao recusarmos o papel de agente da exclusão e da violência institucionalizadas, que desrespeitam os mínimos direitos da pessoa humana, inauguramos um novo compromisso. Temos claro que não basta racionalizar e modernizar os serviços nos quais trabalhamos.

O Estado que gerencia tais serviços é o mesmo que impõe e sustenta os mecanismos de exploração e de produção social da loucura e da violência. O compromisso estabelecido pela luta antimanicomial impõe uma aliança com o movimento popular e a classe trabalhadora organizada.

O manicômio é expressão de uma estrutura, presente nos diversos mecanismos de opressão desse tipo de sociedade. (...)

Contra a mercantilização da doença; contra uma reforma sanitária privatizante e autoritária; por uma reforma sanitária democrática e popular; pela reforma agrária e urbana; pela organização livre e independente dos trabalhadores; pelo direito à sindicalização dos serviços públicos; pelo Dia Nacional de Luta Antimanicomial em 1988 !

Por uma sociedade sem manicômios!

Bauru, dezembro de 1987 - II Congresso Nacional de Trabalhadores em Saúde Mental.

Si en Bauru se introduce la dimensión política y participativa, en la II Conferencia Nacional de Salud Mental (1992), que reúne más de 1500 delegados de Conferencias estatales, asociaciones de profesionales, de familiares y una amplia representación de sufridores psíquicos (cerca del 20\%), se debate durante tres días las ideas del plan para la reforma de la atención la salud mental. La Conferencia, que muestra la fuerza del movimiento reformista y el compromiso del equipo directivo de la salud mental, tanto a nivel federal como de los estados y buena parte de los ayuntamientos, 


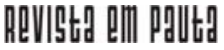

\} SOBRE LA VIGENCIA DEL MANIFIESTO - DESVIAT, M. \}

DOI: $10.12957 /$ REP.2022.63530

supone la consolidación del modelo psicosocial, frente al modelo de asistencia psiquiátrica hospitalocentrista, y la construcción de un programa de salud mental en el ámbito del SUS, de la Reforma Sanitaria. El modelo contempla el territorio como escenario principal de su actuación, un territorio que se encuentra más allá del espejismo unitario de la comunidad, heterogéneo, desigual, intolerante e igualmente solidario, un espacio al cual se incorporan (forman parte) los centros de atención ambulatoria (Caps) y las residencias terapéuticas, pero también las unidades de internamiento de los hospitales generales, las viviendas protegidas y los centros de convivencia entre otra multiplicidad de recursos. Es una red que se plantea enlazada con la atención primaria (Estrategia de Salud de la Familia), puerta de entrada del cuidado de la salud (fig. 1). Propuestas que fueron vinculantes en el programa ministerial que contemplaban modificaciones presupuestarias que lo has harían posible, entre otras, el traspaso del dinero hospitalario a la desinstitucionalización. O propuestas de control de la asistencia que se concretarán posteriormente, como fue la creación del Grupo de Acompañamiento de la Asistencia Psiquiátrica, formado por profesionales externos elegidos en las asociaciones profesionales y de usuarios y familiares (DESVIAT, 2015).

Eduardo Vasconcelos, en un texto publicado en 2016, hace una periodización del proceso de reforma destacando cuatro grandes fases: una primera que sitúa entre 1978 a 1992, con la I Conferencia Nacional de Salud (1986), la emergencia del movimiento antimanicomial (1987) y las primeras experiencias de cambio (Santos, São Paulo, Porto Alegre, Queixada, Betim...); un segundo periodo (1992-2001), durante el cual se da la II Conferencia de Salud Mental citada; un tercer periodo, de 2001 a 2010, cuando sobresale la promulgación de la ley $\mathrm{n}^{-}$10.216, que define las políticas de salud mental de la Reforma, afianzando los derechos de las personas con sufrimiento psíquico. La ley también establece la extinción progresiva de los hospitales psiquiátricos, a ser sustituidos por una red de servicios comunitarios en los cuales se entienda el cuidado en libertad como elemento fundamentalmente terapéutico. En este periodo hay un gran crecimiento de dispositivos y programas de la red psicosocial.

Vasconcelos señala una cuarta fase a partir de 2010, marcada por la recesión económica mundial de finales de la década y por los recortes en las políticas sociales, regida por una creciente crisis política y ética, corrupción sistémica, judicialización partidaria por parte de los sectores más conservadores dirigida contra las fuerzas políticas de centro-izquierda (VASCONCELOS, 2016), consecuente incremento del malestar de la ciudadanía por el debilitamiento de las políticas sociales, con protestas y manifestaciones puntuales que desgastan el gobierno. Esa crisis política y social se agrava en 2015 y 2016, dejando vía libre a un "golpe político blanco" (VASCONCELOS, 2016). En realidad, la tensión social y la situación de derechización de la política mundial reavivaron planteamientos autoritarios 


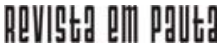

\} SOBRE LA VIGENCIA DEL MANIFIESTO - DESVIAT, M. \}

DOI: $10.12957 /$ REP.2022.63530

y antidemocráticos, tendencias golpistas enmascaradas tras el fin de la dictadura militar, que vieron abrir un escenario de posibilidades.

Por otra parte, hay que tener en cuenta que la reforma sanitaria y psiquiátrica brasileña fue tremendamente desigual en su implementación en los distintos estados y ciudades, como también lo fue el apoyo de la población civil, el enganche civil con los movimientos de la Luta Antimanicomial. En especial, la propagación de programas tan relevantes como Volta para Casa y los centros de convivencia y de generación de renta, fundamentalmente en la última década (ONOCKO-CAMPOS, 2019).

Si es verdad que en la etapa del gobierno de Lula hubo una multiplicación de nuevos servicios, contratación de trabajadoras y la creación de cursos para capacitación en salud comunitaria, producción didáctica por parte del Ministerio de la Salud, también lo es que el SUS no llegó a dar cobertura a la totalidad de la población, siendo muy deficitario en la atención primaria, tan importante para la salud mental, además de haber sido muy precario en los trastornos psíquicos comunes. Eso ha conllevado la existencia de un porcentaje elevado de personas con planes privados de salud, que, en las grandes ciudades, como es el caso de Rio de Janeiro, llega a más del 50\%. (FAGUNDES; DESVIAT; FAGUNDES, 2016). Asimismo, tampoco se llegaron a extinguir los manicomios, manteniéndose los hospitales psiquiátricos penitenciarios.

Digamos que la reforma quedó inconclusa, por lo que es necesario tener en cuenta logros, errores e insuficiencias para su reanudación, muchas de ellas ya apuntadas por autores comprometidos con la reforma: Vasconcelos, Amarante, Pedro Gabriel Delgado, Ana Pitta, Nunes, Bleicher, Onockro, Jackson, Fagundes, Desviat, Venturini, entre otros muchos.

Sin embargo, sin ignorar ese análisis, la cuestión se plantea en términos políticamente simples: la supervivencia del modelo, algo que está estrechamente ligado a la supervivencia de la democracia y la derrota del fascismo del gobierno Bolsonaro. De nuevo el símbolo de este frente de lucha fue Bauru. Consejos federales de diversas categorías profesionales, movimientos de usuarios y de trabajadores, más de 2000 personas, se dieron cita en Bauru a los 30 años del primer encuentro, ocupando de nuevo las calles de esta ciudad, emblema de la Reforma Psicosocial, en los días 8 y 9 de diciembre de 2017, en defensa de los mismos derechos que antaño: la salud, la democracia y la vida.

\section{Algunas conclusiones}

Constaba en Coabitar a diferença (DESVIAT, 2018) que la Reforma Psiquiátrica, cuyo primer objetivo fue sacar a los pacientes mentales de los hospitales psiquiátricos, de los manicomios, y situar servicios de atención en la comunidad, hizo visible la locura y con ella la intolerancia, el estigma, 


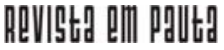

\} SOBRE LA VIGENCIA DEL MANIFIESTO - DESVIAT, M. \}

DOI: $10.12957 /$ REP.2022.63530

la exclusión de la diferencia. Hizo ver que el proceso desinstitucionalizador atravesaba toda la formación social, desvelando prejuicios y representaciones sociales que iban mucho más allá del trastorno psíquico y que situaban a los sufridores psíquicos junto con otros de la exclusión social. Destapó la parte oculta en nuestra sociedad por la dictadura de la Razón, de la podredumbre de la razón, en palabras de Antonin Artaud (1959), en la que los locos son las víctimas por excelencia en un imaginario colectivo poblado de los mitos, las leyendas y los sueños que nos constituyen. Nos acerca a lo que en verdad teje el síntoma singular y social, pues el síntoma se forja en la historia colectiva, en los deseos y miedos ubicados en la trastienda de nuestra cultura. Un proceso que enfrenta a la Reforma de la Salud Mental con la miseria social y subjetiva, donde la acción terapéutica, necesariamente experta en los entresijos técnicos de la terapia y el cuidado, se colorea políticamente.

Dos consecutivas catástrofes, acontecimientos inmersos en la totalidad de la sociedad, han hecho temblar los pilares del sistema político financiero en poco más de una década, mostrando el fracaso del modelo civilizatorio en el que vivimos. En la Gran Recesión, el colapso económico se resolvió con políticas de austeridad en lo servicios públicos, el apoyo de los Estados a los bancos con dinero público y medidas contenedoras de la protesta social que erosionaron la legitimidad democrática y favorecieron el crecimiento de la extrema derecha en todo el mundo, al tiempo que aumentaban el número de multimillonarios y desmedidamente la desigualdad, la precariedad y la pobreza. No repuestos aún de esta catástrofe, una epidemia vírica, altamente predecible, convertida en sindemia por la ineptitud del sistema político-económico mundial para prevenirla y hacerle frente, ha mostrado la fragilidad de los sistemas sanitarios, de la salud pública y de seguridad social de los que hacían gala de los países más desarrollados, colapsando no solo la sanidad, sino la sociedad toda, mostrándoles incapaces de garantizar la protección de la vida, en especial de las poblaciones más débiles, desvelando que la verdadera confrontación que está entre el Capital y la Vida.

La pandemia de la Covid-19 lo ha mostrado claramente: las medidas frente a la pandemia se han planteado en todo el mundo como un conflicto entre la economía y la salud, lo que viene a ser un conflicto entre el capital y la vida, se ha dicho que buscando un equilibrio. En realidad, la vida, sobre todo de las personas más frágiles, ha sido secundaria a la economía.

El viraje sanitario desde las últimas décadas del siglo pasado en beneficio de una sanidad centrada en complejos empresariales hospitalarios tecno-fármaco dependientes, en manos de fondos buitres donde prima la ganancia, pone en peligro a la sociedad toda, en cuanto la privatización, en su priorización de la rentabilidad, ha descuidado la atención primaria e ignorado la salud pública y la epidemiología comunitaria que la podía prevenir a tiempo y atenderla con criterios salubristas, no sólo hospitalarios. 
Figura 1. Red de atención a la salud mental.

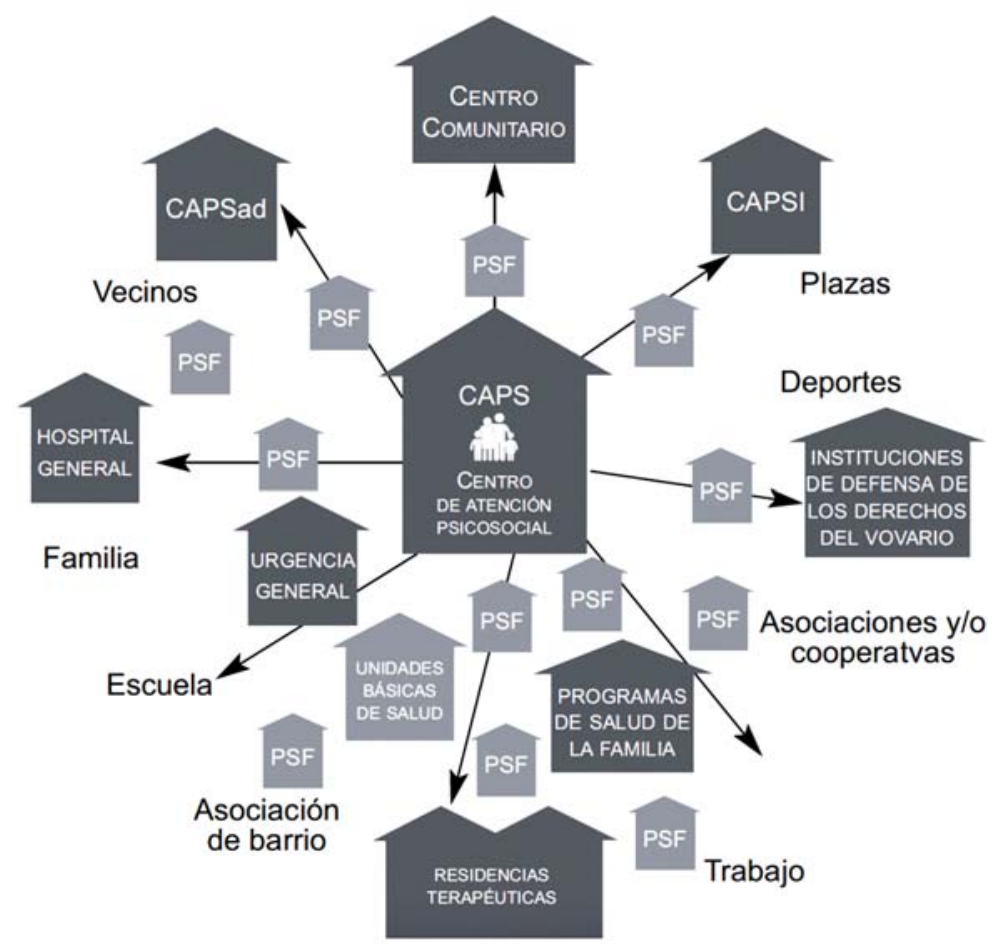

Fuente: AMARANTE, P. Salud mental y atención psicosocial. Madrid: Grupo 5, 2015.

La pandemia muestra dramáticamente las consecuencias de la política del neoliberalismo, depredadora del ecosistema, y desnuda su capacidad para garantizar condiciones dignas de vida, incluso de la vida misma, para una gran mayoría de la población mundial. Mas por muy trágica que pueda ser la pandemia de Covid-19 en el mundo, en Brasil adquiere siniestras peculiaridades, pues, ¿qué se puede aducir cuándo el propio presidente da República niega su existencia y adopta una política de exterminio de la población? ¿Qué respuesta se puede dar con un sistema público de salud infradotado? ¿Con unos equipos de atención primaria prácticamente inexistentes? ¿Con una red de trabajadores que no tienen condiciones adecuadas de trabajo? ¿Con un gobierno que invierte en instituciones cerradas, como los manicomios y las comunidades de atención a los usuarios de alcohol y drogas, potenciadores del contagio por la Covid-19? ¿Con mecanismos de controle social ausentes o fragilizados? (BLEICHER, 2020).

Brasil desnuda la política de la extrema derecha, esa nueva derecha neoliberal que no contempla la solidaridad ni la compasión; que, frente 


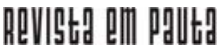

\} SOBRE LA VIGENCIA DEL MANIFIESTO - DESVIAT, M. \}

DOI: $10.12957 /$ REP.2022.63530

al nosotros de lo común, plantea un otro como enemigo en quien descargar el malestar social que ocasiona, ya sean los emigrantes, los negros, los indígenas, los comunistas, los nadie...

El Brasil de Bolsonaro es un ensayo, una vía a tener en cuenta para el desarrollo del capitalismo del siglo XXI, como lo es la América de Trump o lo fue el Chile de Allende para el neoliberalismo del siglo XX. Un escenario posible para un sistema que hace tiempo da señales de inestabilidad, social, ambiental, económica. De hecho, tras la crisis financiera del 2008 y la sindemia de la Covid-19, no solo se ha perdido la fe en el progreso o la simple confianza en una cierta mejora progresiva de las condiciones de vida: se ha llegado a temer el colapso financiero y político del sistema que gobierna el mundo y hasta a cuestionar la supervivencia de vida de nuestra especie en el planeta. La situación descrita por el colectivo de cineastas galos Les Parasites en la serie televisiva Colapso no parece ya algo de ciencia ficción. Como tampoco lo son la consideración del sociólogo Boaventura Sousa Santos (2013), cuando dice que si se sigue defendiendo que no hay alternativa posible, ello nos conduce al suicidio. 


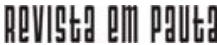

\} SOBRE LA VIGENCIA DEL MANIFIESTO - DESVIAT, M. \}

DOI: $10.12957 /$ REP.2022.63530

\section{Referências}

AMARANTE, P. Salud mental y atención psicosocial. Madrid: Grupo 5, 2015. ARTAUD, A. Carta a los poderes. Buenos Aires: Mundo Nuevo, 1959.

BLEICHER T. Rede de Atenção Psicossocial antes, durante e após a pandemia de Covid-19. In: SOUSA FÉLIX T. (Ed.). Politicas públicas no Brasil: incursões na saúde, educação e psicologia. Curitiba: CRV, 2020.

CRUZ, N.; GONÇALVES, R.; DELGADO, P. Retrocesso da reforma psiquiátrica: o desmonte da política nacional de saúde mental brasileira de 2016 a 2019. Trabalho, Educação e Saúde, v. 18, n. 3, 2020. Disponível em: https:/ /doi. org/DOI: 10.1590/1981-7746-sol00285. Acesso em: 12 nov. 2021.

DESVIAT, M. (Neo)capitalismo e sofrimento psíquico. Revista Movimento, 23 dez. 2019. Disponível em: https://movimentorevista.com.br/2019/12/ neocapitalismo-e-sofrimento-psiquico/. Acesso em 12/11/2021.

DESVIAT, M. A reforma psiquátrica. Rio de Janeiro: Fiocruz, 2015.

DESVIAT, M. Coabitar a diferença. Da reforma psiquiátrica à saúde coletiva. São Paulo: Zagodoni, 2018.

FAGUNDES, H.; DESVIAT, M.; FAGUNDES, P. Reforma psiquiátrica no Rio de Janeiro: situação atual e perspectivas futuras. Ciência e Saúde Colectiva, v. 21, n. 5, 2016. Disponível em: https://doi.org/10.1590/141381232015215.00872016. Acesso em: 12 nov. 2021.

FAGUNDES, S. Águas da pedagogia da implicação: intercessões da eduação para políticas públicas de saúde. Rio Grande do Sul: UFRGS, 2006. Disponível em: https://www.lume.ufrgs.br/handle/10183/16185. Acesso em: 12 nov. 2021.

MANIFESTO de Bauru. In: CONGRESSO NACIONAL DE TRABALHADORES DA SAÚDE MENTAL, 2, Bauru, 1987. Disponível em: http://site.cfp.org.br/ wp-content/uploads/2017/05/manifesto-de-bauru.pdf. Acesso em: 12 nov. 2021.

ONOCKO-CAMPOS, R. Salud mental en Brasil: avances, retrocesos y desafíos. Cad. Saúde Pública, v. 35, n. 11, 2019. Disponível em: https://www.scielo.br/ j/csp/a/LKMxbhKYbPHqP8snJjHwsLQ/?lang=pt. Acesso em: 12 nov. 2021. CARVALHO, S. Saúde colectiva e promoção da saúde. São Paulo: Hucitec, 2007. SANTOS, B. S. Palavras. 1 dez. 2013.

VASCONCELOS, E. Reforma psiquiátrica, tempos sombrios e resistência: diálogos com o marxismo e o serviço social. Campinas: Papel Social, 2016.

DOI: 10.12957/rep.2022.63530

Recebido em 08 de outubro de 2021. Aprovado para publicação em 17 de outubro de 2021.

A Revista Em Pauta: Teoria Social e Realidade Contemporânea está licenciada com uma Licença Creative Commons Atribuição 4.0 Internacional. 